\title{
Circular Differential Scattering of Single Chiral Self-Assembled Gold Nanorod Dimers
}

\author{
Lin-Yung Wang ${ }^{\dagger, \ddagger, 1}$, Kyle W. Smith ${ }^{\dagger, \ddagger, 1}$, Sergio Dominiquez-Medina $a^{\dagger, \ddagger}$ \\ Nicole Moody ${ }^{\dagger, \ddagger}$, Jana M. Olson ${ }^{\dagger, \ddagger}$, Huanan Zhang ${ }^{\|}$, Wei-Shun Chang ${ }^{*}{ }^{,}, \ddagger$, \\ Nicholas Kotov*, ${ }^{*}$, Stephan Link ${ }^{*},+, \ddagger, \S$ \\ ${ }^{\dagger}$ Department of Chemistry, Rice University, 6100 Main Street Houston, TX 77005, USA \\ ${ }^{\ddagger}$ Laboratory for Nanophotonics, Rice University, 6100 Main St. Houston, TX, 77005, USA \\ ${ }^{\S}$ Department of Electrical and Computer Engineering, Rice University, 6100 Main St., \\ Houston, TX, 77005, USA \\ "Department of Chemical Engineering, University of Michigan, 2300 Hayward St., Ann \\ Arbor, MI 48109 \\ ${ }^{1}$ These authors contributed equally \\ *Address corresponding to \\ slink@rice.edu, \\ kotov@umich.edu, \\ wschang@rice.edu,
}




\begin{abstract}
Circular dichroism measures the differential interaction of matter with left-handed and righthanded circularly polarized light. Circular dichroism spectroscopy is essential for quantifying protein secondary structure composition, and has more recently been employed to probe chiral nanomaterials, which can have extraordinary strong circular dichroism effects compared to their molecular counterparts. Here we investigated the circular differential scattering of a simple chiral plasmonic system, a twisted side-by-side Au nanorod dimer, using single-particle circular dichroism spectroscopy complimented with electromagnetic simulations. This approach enabled us to quantify the effects of structural symmetry breaking, namely size-mismatch between the constituent Au nanorods and large twist angles, on the resulting circular dichroism scattering spectrum. Our results demonstrate that, if only scattering is considered as measured by dark-field spectroscopy, a homo-dimer of Au nanorods with similar sizes produces a circular differential scattering lineshape that is different from the bisignate response of the corresponding conventional CD spectrum, which measures extinction for this nanorod dimer sample, i.e. the sum of scattering and absorption. On the other hand, symmetry breaking in a hetero-dimer with Au nanorods with different sizes yields a bisignate circular differential scattering lineshape. In addition, we provide a general method for correcting linear dichroism artifacts arising from slightly elliptically polarized light in a typical dark-field microscope, as is necessary especially when measuring highly anisotropic nanostructures, such as side-by-side nanorods. This work lays the foundation for understanding absorption and scattering contributions to the CD lineshape of single nanostructures free from ensemble-averaging, especially important for self-assembled chiral nanostructures that usually exist as both enantiomers.
\end{abstract}


Keywords: Chirality, nanoparticle, surface plasmon, self-assembly, single-particle spectroscopy

TOC Graphic

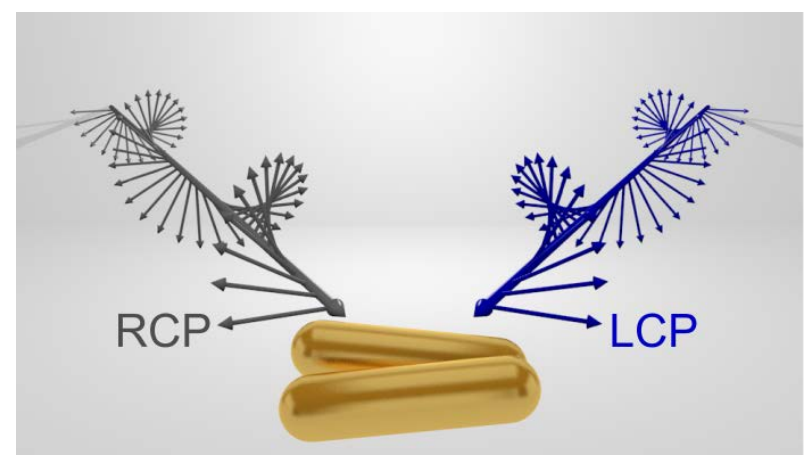

Chiral molecules, asymmetric chemical structures possessing non-superimposable mirror images, ${ }^{1}$ exhibit stereoselectivity in chemical reactions and biological interactions. ${ }^{2}{ }^{3}$ They are also the building blocks of life, imparting chirality on macrobiological structures of various conformations. ${ }^{4,5}$ In both natural and synthetic stereoselective reactions, the use of one chiral molecule rather than its enantiomer can result in very different yields. Similarly, some enantiomers of proven drugs are poisonous. ${ }^{6}$ Therefore it is crucial to accurately determine molecular chirality, which is usually quantified by circular dichroism (CD), the differential optical response of a system interacting with a left-handed circularly polarized (LCP) and a righthanded circularly polarized (RCP) light. ${ }^{7}$ CD spectroscopy is extensively used in the field of biochemistry as it offers qualitative (folded vs. unfolded) and quantitative (percent compositions of $\alpha$-helix, $\beta$-sheet, random coils, etc.) insight into protein conformations, ${ }^{8}$ sugar binding, ${ }^{9}$ and DNA base-base interactions. ${ }^{10}$

In typical measurements of chiroptical activity, differential extinction signals are acquired on ensembles of (macro-)molecules and the resulting CD lineshape is composed of both positive and negative bands, due to the Cotton effect. ${ }^{11}$ Energy conservation requires that the overall integrated extinction CD spectrum is zero. ${ }^{12}$ However, such energy conservation does not 
apply when absorption and scattering signal are measured separately. ${ }^{12,}{ }^{13}$ This distinction between scattering and absorption vs. their sum, i.e. extinction, is especially important as the size of the chiral object increases from a small molecule, for which scattering can often be completely neglected. However, the scattering cross sections of macromolecules such as proteins can be comparable to or even larger than their absorption cross sections. Bustamante et al. elegantly demonstrated in 1983, both theoretically and experimentally, that the scattering contribution to the CD response is non-negligible when the size of the macromolecule reaches $1 / 20$ th the wavelength of the incident light. ${ }^{13}$ They also reported a dramatic change in the lineshape of the circular differential scattering (CDS) spectrum, which exhibits only one band of a certain sign, while the corresponding absorption CD spectrum possesses a bisignate lineshape. ${ }^{13}$ This fundamental difference is often neglected due to the overwhelming success in the technically less demanding extinction-based ensemble CD measurements.

Recently, ensemble CD spectroscopy has been used to characterize chiral nanomaterials, ${ }^{14-36}$ which are of interest as negative refraction metamaterials, ${ }^{25}$ miniaturized optical elements with giant chiroptical effects, ${ }^{26,27}$ and enhanced (bio-)sensing platforms. ${ }^{28,} 30$ New chiral nanomaterials have been developed through novel top-down fabrication 21, 24, 26, 31-33 and bottom-up synthesis 15, 18-20, 22, 23, 34-37 methods. These nanomaterials are generally large enough that scattering cannot be neglected. Especially for plasmonic nanostructures treating absorption and scattering separately is important as one determines heat generation or hot electron production ${ }^{38}$ and the other is probes the radiative strength of the surface plasmon. ${ }^{39}$

The single-particle technique most widely used for plasmonic nanostructures, dark-field spectroscopy, ${ }^{40}$ exclusively measures scattering. Other techniques such as spectral interference microscope, $^{41}$ spatial modulation spectroscopy, ${ }^{42}$ super-continuum white light confocal 
microscopy, ${ }^{43}$ and photothermal imaging ${ }^{44}, 45$ were developed to probe the extinction or absorption of single nanoparticle. Single-particle techniques are particularly important for studying chiral nanostructures prepared by self-assembly techniques that enable the rapid formation of three-dimensional objects with small interparticle gaps and hence strong plasmonic coupling, ${ }^{46}$ but are inherently prone to introduce heterogeneity. Single-particle studies on heterogeneous samples enable subtle structural features to be directly related to changes in spectral properties. ${ }^{47}$ However, despite the otherwise successful use of single-particle spectroscopy very few studies exist on the CD response of chiral plasmonic nanostructures. ${ }^{16,17}$, 24, 34, 48 Among these few reports, elegant CD spectroscopy using non-linear spectroscopy ${ }^{17,23,49}$ and near-field microscopy ${ }^{24}$ did not yield either pure absorption or scattering, while CD spectra measured in a dark-field scattering microscope ${ }^{48}$ resulted from achiral nanostructures due to the geometry created by oblique angle excitation.

Here, we demonstrate on side-by-side twisted Au nanorod (AuNR) dimers, one of the simplest assembled chiral plasmonic nanostructures, that, similar to proteins, the CDS spectrum of AuNR dimers is composed of only one band. However, symmetry breaking results in a large change of the CDS spectrum. Finite-Difference Time Domain (FDTD) simulations reveal that the appearance of a bisignate lineshape is mainly due to the size mismatch of the two constituent AuNRs and an increase in twist angle $\theta$.

\section{Results and Discussion}

When two identical AuNRs are placed side-by-side, the resulting "homo-dimer" is chiral if the twist angle $\theta$ between the AuNRs is between $0^{\circ}$ and $90^{\circ}\left(\mathrm{D}_{2}\right.$ point group, Figure 1a). A mirror plane exists though in the overall structure for $\theta=0^{0}$ or $90^{\circ}\left(\mathrm{D}_{2 \mathrm{~h}}\right.$ and $\mathrm{D}_{2 \mathrm{~d}}$ point groups, respectively). Here we always refer to the "AuNR dimer" as the chiral side-by-side twisted 
AuNR dimer. Although the determination of the chirality from the dimer geometry is a simple 'true-false' question, the spectroscopic CD response is far more complex.
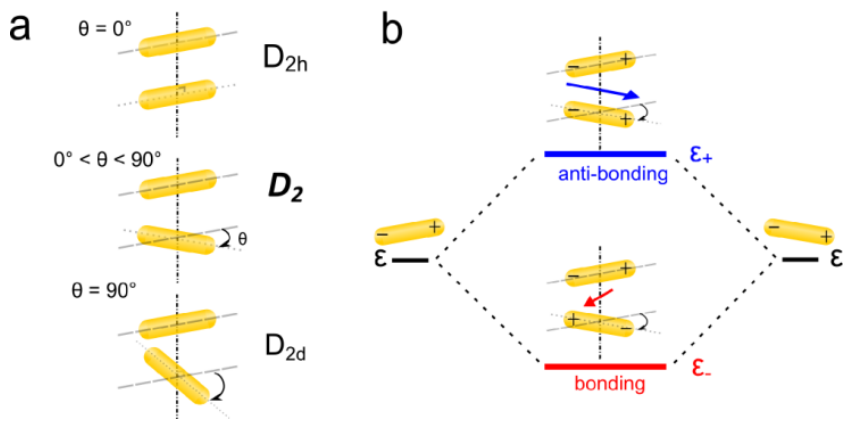

Figure 1. Geometries of twisted AuNR dimers. (a) Illustrations of twisted AuNR dimers with 3 representative twist angles $\theta$ resulting in the associated point groups shown. Twist angle $\theta$ is defined by the angle difference between two AuNRs where the bottom rod is rotated by $\theta$ around the twist axis which is perpendicular to the long axes of two AuNRs as indicated by the dashed line. (b) Plasmon hybridization diagram for the side-by-side AuNR dimer system. The plus and minus signs denote the charge of the plasmon oscillation and the arrows represent the directions of the overall dipole moment.

Plasmon hybridization theory, ${ }^{50,51}$ the plasmonic analogue of molecular orbital theory, offers intuitive predictions of the AuNR dimer CD response. ${ }^{12,32}$ By ignoring contributions from coupling of transverse and higher order modes, each AuNR can be approximated by only considering its longitudinal mode as a dipole with energy $\varepsilon$ (Figure 1b). When the AuNRs are close enough to couple, the degenerate energy levels split into a lower energy bonding mode $\varepsilon$ and a higher energy anti-bonding mode $\varepsilon_{+}$(Figure 1b). For the bonding mode, the dipoles are aligned in an anti-parallel manner, therefore relieving the repulsion of charge and resulting in a lower energy. Because the dipoles nearly cancel out in this configuration, this lower energy bonding mode has a small transition dipole moment and is mostly optically dark. On the other hand, for the anti-bonding mode, the parallel alignment of dipoles gives rise to a higher energy mode because of charge repulsion. As the dipoles add up constructively in this configuration though, the anti-bonding mode can be excited optically. It is important to note that the antibonding mode has a handedness that is the same as the handedness of the AuNR dimer structure, 
while the bonding mode has an opposite handedness, resulting in the two modes possessing CD responses of opposite sign. ${ }^{32}$

The twisted AuNR dimer system has been investigated theoretically before using a coupled dipole theory. ${ }^{12}$ We have applied full-wave electromagnetic simulations using the FDTD method and approximating the AuNRs as cylinders capped with hemispheres instead of ellipsoids, and have obtained similar results in the limit of large interrod distances. However, for small AuNR separations of a few $\mathrm{nm}$ as is the case in the experiments discussed below, fullwave electromagnetic simulations are necessary to correctly describe this system (Figure S2). Calculated absorption cross sections $\sigma_{a b s}$ as a function of wavelength, i.e. absorption spectra, of a AuNR dimer with a positive twist angle for excitation with LCP and RCP light indeed showed two resonance maxima at $843 \mathrm{~nm}$ and $1049 \mathrm{~nm}$, corresponding to the anti-bonding and the bonding modes, respectively (Figures 2 and S1). The charge density plots calculated at these two energies (Figure 2a insets) confirm this mode assignment. At the anti-bonding mode, this AuNR dimer with a positive twist angle (i.e., the (+)-enantiomer) absorbs RCP light stronger compared to LCP light because the twist of the AuNR 'seen' second in the path of light propagation matches with the twist direction of the circular polarization, where the k-vector is parallel to the twist axis. Similarly at the bonding mode, the (+)-enantiomer absorbs stronger under excitation with LCP light. This differential absorption, $\Delta \sigma_{a b s}=\sigma_{a b s}{ }^{L C P}-\sigma_{a b s}{ }^{R C P}$, is illustrated by the absorption CD spectrum in Figure 2b. The absorption CD spectrum displays the typical bisignate lineshape associated with molecular CD spectra: one negative band at the anti-bonding mode and one positive band at the bonding mode as indicated by the two dotted lines. The CD spectrum of the same dimer with the opposite twist, i.e. the (-)-enantiomer, is the mirror image of that of the (+)-enantiomer. At a first glance, plasmon hybridization theory and the calculated absorption CD 
spectra suggest a strong analogue of the AuNR dimer to the optical response of chiral molecules that have been modeled with exciton coupling theory. ${ }^{7,}{ }^{11}$ We demonstrate in the following paragraph that this analogy is not fully correct though, as scattering also has to be considered, consistent with what has been reported before based on simulations using the coupled dipole approximation. $^{12}$

In contrast to absorption, the scattering spectra of the (+)-enantiomer calculated with excitation using LCP and RCP light show only one resonance, which corresponds to the antibonding mode (Figure 2c). The corresponding scattering CDS spectrum, $\Delta \sigma_{\text {scat }}=\sigma_{\text {scat }}{ }^{L C P}-\sigma_{\text {scat }}{ }^{R C P}$, also has only one band (Figure 2d). The absence of the bonding mode can be explained within the electrostatic dipole limit considering that the scattering cross section is proportional to the square of the polarizability $\alpha$, i.e. $\sigma_{\text {scat }}=k^{4} / 6 \pi|\alpha|^{2}$ where $k$ is the wave vector. For the bonding mode, the anti-parallel alignment of the two AuNR dipoles results in a small value for the square of the polarizability, resulting in a negligible contribution to both scattering and CDS spectra. ${ }^{12}$ Unlike scattering, absorption has only a linear dependence on the imaginary part of the polarizability, i.e. $\sigma_{a b s}=k \cdot \operatorname{Im}[\alpha]$. Therefore the small residual dipole of the anti-bonding mode appears in the absorption spectra and especially in the absorption CD spectra. 


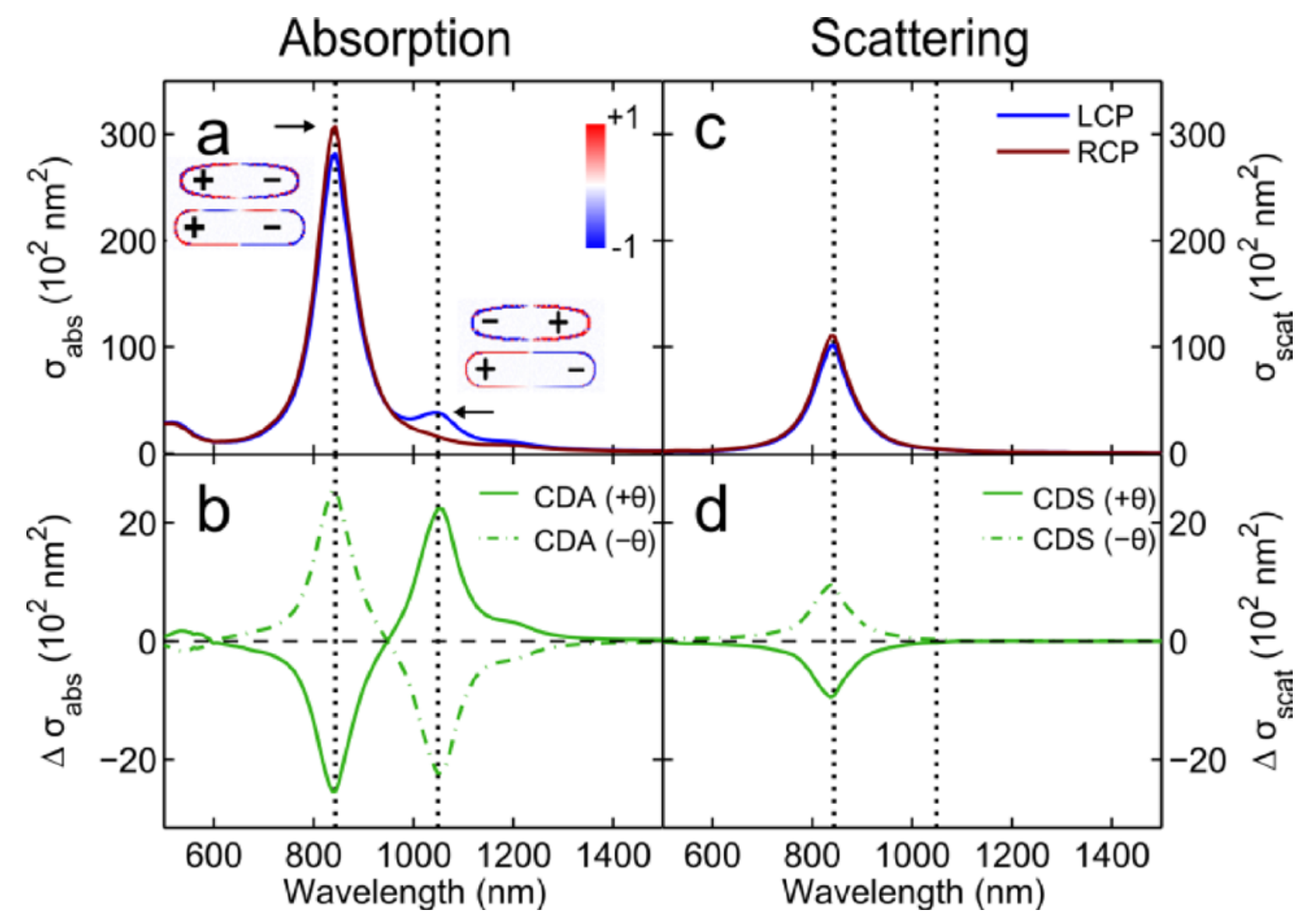

Figure 2. Calculated scattering, absorption and their differential spectra of twisted AuNRs dimers. FDTD simulations of the absorption spectra (a), absorption CD spectra (b), scattering spectra (c), and CDS spectra (d) of dimers with positive and negative twist angles (for clarity only scattering spectra of the $+\theta$ enantiomer are shown in (a) and (c)). Inset: Charge plots shown in (a) were calculated at $843 \mathrm{~nm}$ and $1049 \mathrm{~nm}$ under LCP excitation, revealing the anti-bonding and bonding nature of these resonances. The color bar is the corresponding relative charge density scale. The elliptical shape of the top nanorods results from the fact that the plane of the cross-section contained the bottom nanorods. The width and length of both AuNRs were 22 and $88 \mathrm{~nm}$, respectively. The gap between the AuNRs was $8 \mathrm{~nm}$ with a twist angle of $\theta=+16^{\circ}$. See Methods Section and Figure S1 for further details.

One of the characteristics of CD spectra is the sum rule. For molecular CD spectroscopy, it has been theoretically proven that the integrated CD signal is zero as a result of energy conservation. ${ }^{11,12,52}$ The fact that only one band is observed in the CDS spectra (Figure 2d) does not imply that this sum rule breaks down though. When the CD signal of the total attenuated energy - the sum of absorption and scattering - is considered for the AuNR dimer, we find that for $\Delta \sigma_{\text {ext }}=\Delta \sigma_{\text {scat }}+\Delta \sigma_{a b s}$ the sum rule still applies. The drastic difference in the CDS lineshape 
has been demonstrated in early work by Bustamante et al. on proteins using ensemble circular intensity differential scattering techniques. ${ }^{13}$ However, little attention has been paid to this effect for chiral plasmonic nanostructures, mostly because ensemble CD spectra measure generally the extinction.

We collected single-particle CDS spectra in order to test this theoretical model and also to understand the role of size mismatch in 'real' AuNR dimers. We first had to establish a robust procedure for how to measure the CDS spectra of individual nanostructures free from polarization artifacts introduced by a traditional dark-field microscope. The incident light in our transmitted-light microscope was circularly polarized and switchable between LCP and RCP using a combination of a linear polarizer and a quarter-waveplate (Figure 3a). LCP and RCP light passed through an oil immersion dark-field condenser with an incident angle of $69^{\circ}$ which was larger than the critical angle at the glass-air interface to generate an evanescent wave (Figure S3).$^{53}$ The dark-field condenser furthermore ensured that the excitation light was incident from all $360^{\circ}$ directions (Scheme S1), resulting in an in-plane averaged CDS spectrum. This excitation geometry avoided contributions from an extrinsic CDS signal of achiral nanoparticles as reported recently. ${ }^{48}$ These in-plane averaged measurements are not directly comparable to ensemble measurements which necessarily average over all possible incident directions in three dimensions, but simulations for this particular AuNR dimer system suggest that the difference between in-plane averaged and 3D excitation does not change the lineshape of the CDS spectrum (Figure S4). 


\section{a}

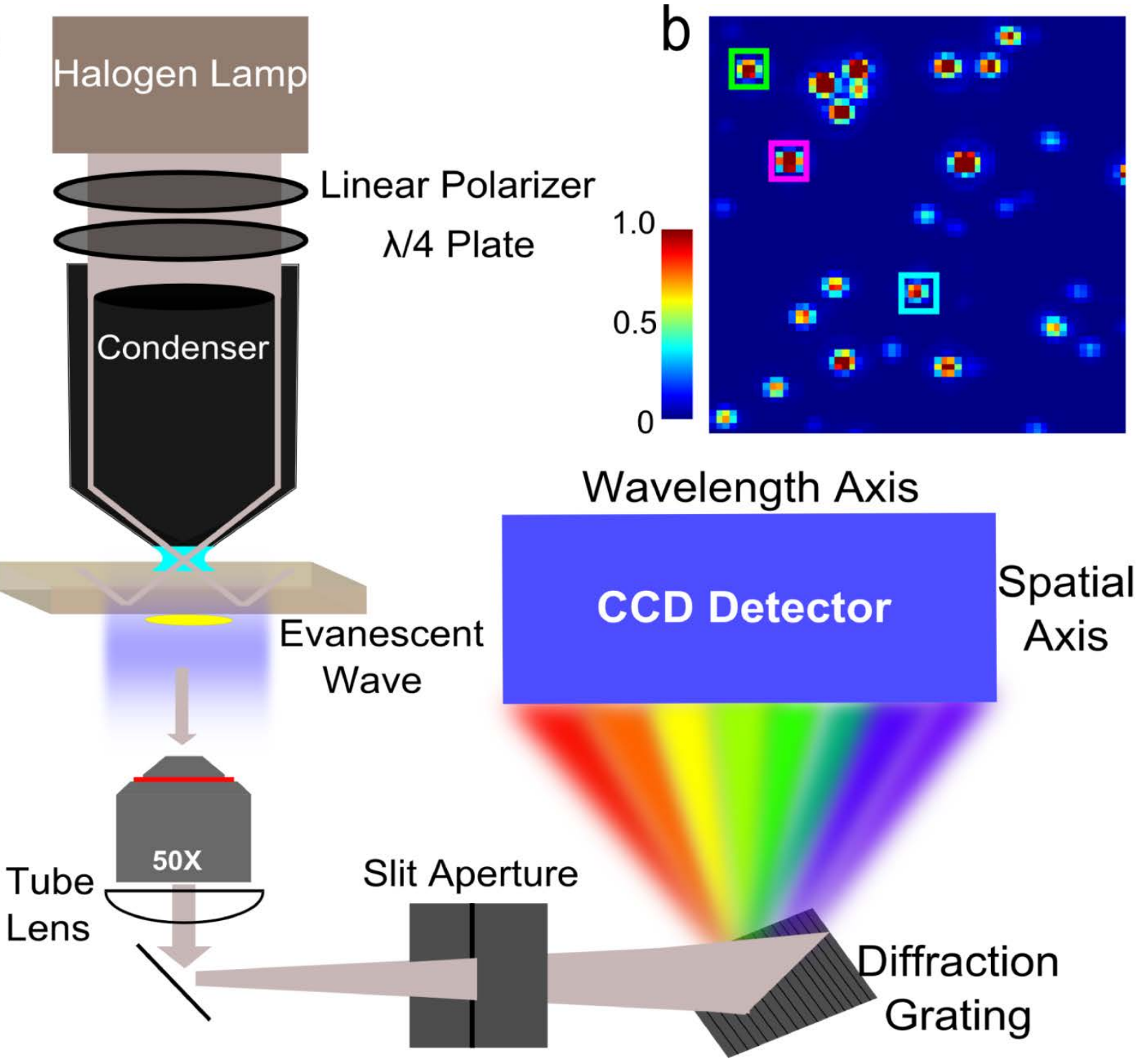

Figure 3. Experimental single-particle CDS setup. (a) Instrument diagram of the inverted, darkfield microscope adapted to collect scattering CDS spectra of single nanoparticles. (b) A representative hyperspectral image of single AuNRs with three AuNRs identified by colored boxes for correlation to their spectra in Figure 4.

The scattered light of the nanoparticles was collected by an objective and directed to a hyperspectral detection system (Figure 3b and Methods Section) for the rapid collection of spectra from a large numbers of nanoparticles, particular useful for the correction of linear dichroism artifacts as discussed below. CDS spectra are reported as a unitless anisotropy factor, Percent $C D S=100 \times\left(2\left(I_{L C P}-I_{R C P}\right) /\left(I_{L C P}+I_{R C P}\right)\right)$, where $I_{L C P}$ and $I_{R C P}$ are the 
wavelength-dependent intensities of the nanoparticle scattering with incident LCP and RCP light, respectively. This normalization allows for the direct comparison of CDS measurements from chiral nanoparticles with significantly different scattering intensities.

We first considered the departure from perfect circular polarized excitation, which is experimentally unfeasible to achieve with a dark-field condenser over a broad wavelength range. It is well-established that CDS measurements of oriented anisotropic samples can lead to linear dichroism artifacts, ${ }^{54,55}$ if even a small degree of ellipticity exists for the circularly polarized excitation. To avoid this artifact, we utilized a highly anisotropic achiral control, namely a single AuNR, to develop a correction procedure. For achiral single AuNRs deposited randomly via spin coating on a glass substrate, we observed a systematic error in the measured CDS (Figure 4a-c) signal as a function of their orientation that was determined from the linear polarization dependence of their scattered light by rotating a polarizer in the detection path (Figure 4d-f). Linear dichroism artifacts manifest themselves in AuNR CDS spectra because slight ellipticity of the incident excitation results in LCP and RCP light having major axes offset relative to each other by $90^{\circ}$ (Scheme S2). The spatial distribution of the polarization of elliptical incident light for the experimental cone excitation is displayed in scheme S1d. The electric field distributions of LCP and RCP excitations averaged over all incident angles in the sample plane therefore show slightly elliptical shapes (Figure 4g). This unequal distribution of the excitation field for the two incident polarization conditions employed in the CDS measurements results in AuNR dipoles of different orientations interacting more strongly with either LCP or RCP light despite the AuNR being achiral (Figure 4h). 

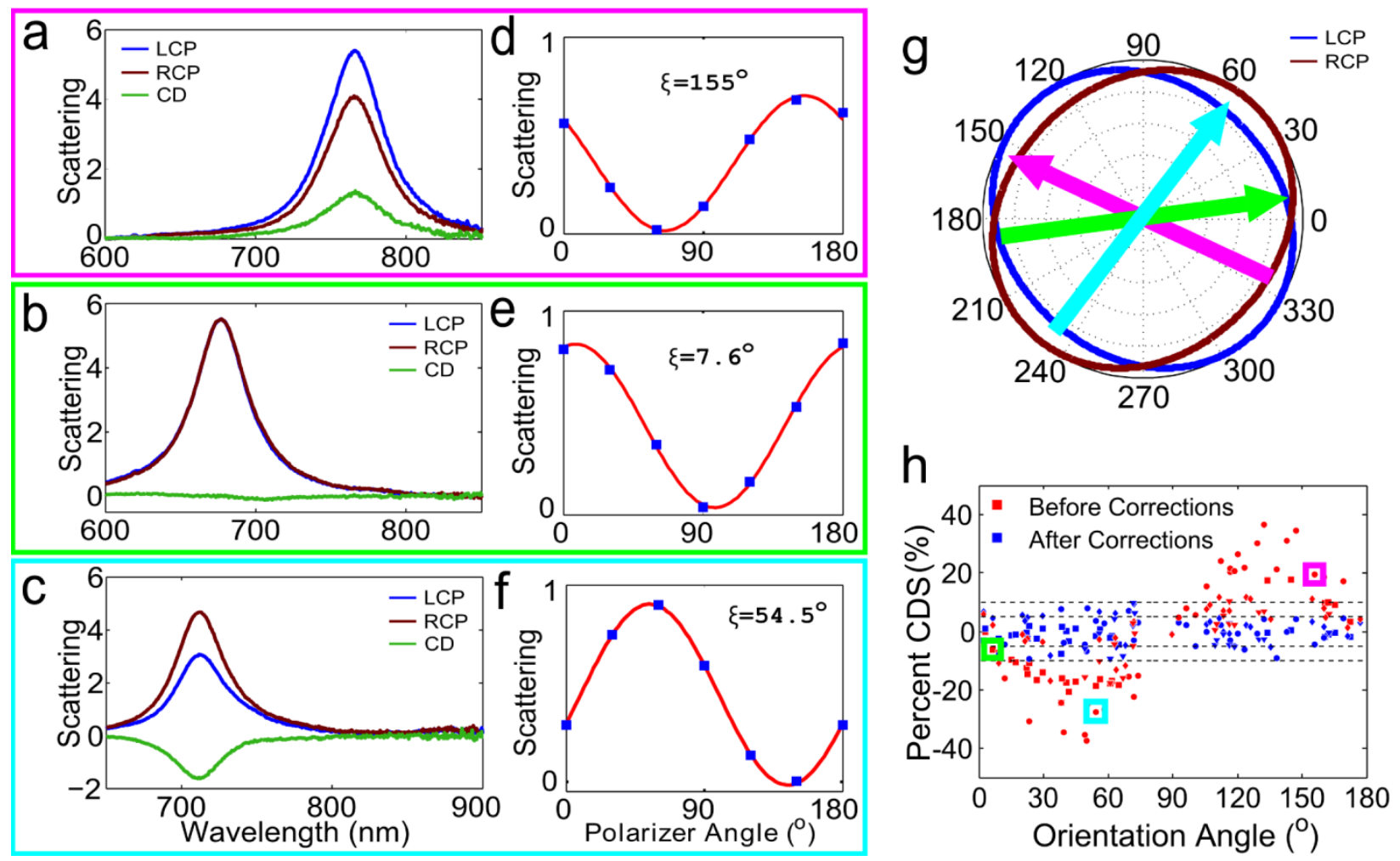

Figure 4. Correction procedure to account for imperfect circular polarization of the excitation light. $(\mathrm{a}-\mathrm{c})$ Scattering spectra of single AuNRs were recorded with incident LCP and RCP light (blue and brown lines, respectively) and yielded the single-particle CDS spectra given by the green lines. $(\mathrm{d}-\mathrm{f})$ Polarization dependence of the scattering intensity at the peak resonance wavelength, collected separately for these individual AuNRs by changing the angle of a polarizer placed in the detection path. This scattering intensity modulation was fit (red line) to determine the AuNR orientation. (g) Schematic representation of the electric field for elliptically polarized light with the dipole orientations of the highlighted AuNRs in (a - c) overlaid. (h) Percent CDS collected from 99 single AuNRs as a function of their orientation before (red) and after a correction factor was applied (blue). Calibration measurements from several days were included in (h). Dashed lines at $\pm 5 \%$ and $\pm 10 \%$ CDS are included as guides for the eye. The data points marked with the colored boxes correspond to the AuNRs highlighted in Figures 3b and 4a-c.

Based on the 'false' CDS spectra of a total of 99 randomly orientated AuNRs, we were able to calculate a correction factor that, when applied to the measured CDS spectra of chiral nanostructures, account for the ellipticity in the incident excitation source. Figure $4 \mathrm{~h}$ plots the measured Percent CDS determined at the plasmon peaks, chosen to fall in the same wavelength range as the AuNR dimers, as a function of AuNR orientation. The phase dependence of the systematic deviation from no CDS signal reflects the orthogonal major axes of elliptical LCP and 
RCP light that preferentially excite AuNRs that are aligned parallel to these axes (Figure 4g). Based on the data in Figure 4h, we developed a wavelength-independent correction factor to account for 'false' CDS signals of achiral nanostructures caused by linear dichroism. Further details regarding this correction procedure are presented in the Supplemental Information (SI). This model reproduces the trend in Figure $4 \mathrm{~g}$ well (Scheme S3), therefore validating our approach. After application of the correction factor, the CDS signal for single AuNRs was reduced to within $10 \%$ of the scattering intensity with no apparent orientation dependence of CDS signal (Figure 4h), justifying our assumption that single AuNR are achiral even though imperfections at the atomic level especially at the AuNR ends could lead to a minor degree of chirality. Accordingly, we used $10 \%$ as our detection limit for the CDS spectra of chiral nanostructures. We furthermore performed FDTD calculations of the CDS for single AuNRs in the presence of a substrate to ensure that symmetry breaking due to a substrate did not induce an artificial CDS signal in our experimental geometry (Figure S5). We stress that this correction factor was not specific to AuNR dimers or even plasmonic nanoparticles, but represents a general procedure that can be applied to single-particle CDS spectroscopy and other excitation geometries. Less anisotropic scatterers can be accounted for by reducing the magnitude of the correction factor according to their anisotropy (SI eq. 22-24). A completely isotropic scatterer requires no application of a correction factor. Measurement of spherical Au nanoparticles which are achiral and approximately isotropic scatterers possessed no CDS signal without the application of a correction factor (Figure S6), supporting our linear dichroism correction method and ensuring systematic errors were not the origin of the CDS artifacts.

We successfully applied our single-particle CDS spectroscopy to individual chiral AuNR dimers. Figure 5a shows the scattering spectra under LCP and RCP light excitation for a single 
dimer of AuNRs with similar lengths (termed as the experimental homo-dimer) as confirmed by the scanning electron microscope (SEM) image shown in the inset. In agreement with the model AuNR dimer discussed in Figure 1, the resulting CDS spectrum (Figure 5b) clearly showed only one negative CDS band above the detection limit, indicating that this homo-dimer had a positive twist angle.

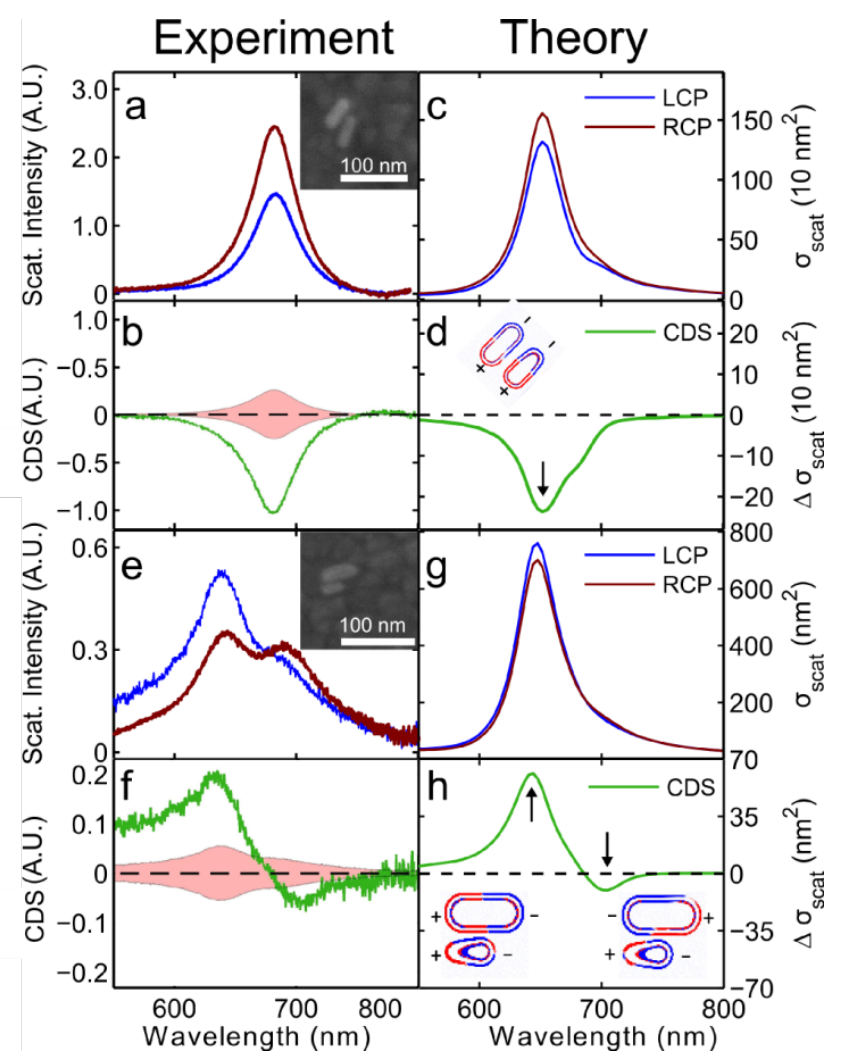

Figure 5. Experimental and calculated scattering and CDS spectra of two AuNR dimers. (a) Scattering spectra recorded with incident LCP and RCP light and the corresponding CDS spectrum (b) of a twisted dimer comprised of two AuNRs with similar widths and lengths (i.e. homo-dimer) as determined by correlated SEM imaging (inset in (a)). The red region in (b) denotes the 10\% detection limit determined from CDS measurements of single AuNRs (Figure 4). Panels (c) and (d) show the corresponding calculated spectra for a dimer comprised of AuNRs with width x length dimensions of $20 \mathrm{~nm}$ x $54 \mathrm{~nm}$ and $20 \mathrm{~nm}$ x $54 \mathrm{~nm}$. The interparticle distance was set to $2 \mathrm{~nm}$ with a twist angle of $\theta=12^{\circ}$. The surface charge distribution calculated at $651 \mathrm{~nm}$ shows that this resonance corresponds to the anti-bonding mode. (e) Scattering spectra recorded with incident LCP and RCP light and the corresponding CDS spectrum (f) of a dimer comprised of two AuNRs with distinctively different widths and lengths (i.e. heterodimer). Guided by the SEM image, simulations for this hetero-dimer shown (g) and (h) were based on AuNRs with width x length dimensions of $28 \mathrm{~nm}$ x $56 \mathrm{~nm}$ and $12 \mathrm{~nm}$ x $35 \mathrm{~nm}$. Best agreement with experiments was achieved with an interparticle distance of $2 \mathrm{~nm}$ and a twist 
angle of $\theta=-45^{\circ}$. These parameters are not directly accessible through the SEM images that only provide two-dimensional projections of the nanostructures. Surface charge distributions calculated with the incident k-vector along the dimer twist axis at the two CDS peaks, $619 \mathrm{~nm}$ and $757 \mathrm{~nm}$, indicate that these two resonances correspond to the anti-bonding and bonding modes, respectively. The simulated CDS spectra were obtained by averaging all in-plane excitation directions (Figure S7). A substrate with a refractive index of $n=1.5$ was included in these calculations. See Methods Section and Figure S8 for further details.

FDTD calculations reproduce the single-particle spectra. To simulate the experimental geometry of the condenser and evanescent wave excitation, the $k$-vector of the excitation light was assumed to be parallel to the substrate and the CDS spectrum was averaged over two orthogonal $k$-vectors directions. ${ }^{56}$ The intensity of the in-plane averaged CDS spectrum $\langle\mathrm{CD}\rangle_{\text {in- }}$ plane was half the magnitude of the simulated CDS for the $k$-vector parallel to the dimer twist axis (Figure S7), as the orthogonal in-plane direction did not interact strongly with the coupled AuNR dimer modes. The AuNR dimensions were determined from the correlated SEM image, and a glass substrate to support the dimers was included (Figure S8). The calculated spectra agree well with the experiment for a twist angle of $12^{\circ}$ (Figure 5c-d). A twist angle of $8^{\circ}$ was reported by ensemble measurements. ${ }^{34}$ The surface charge plot in Figure 5d indicates that the CDS peak at $651 \mathrm{~nm}$ indeed corresponds to the bright anti-bonding mode. The small disagreement for the resonance maxima, which were blue-shifted in the calculations, could have resulted from the limited structural and orientation information of the SEM image.

Significant changes to the predicted single band CDS spectrum were observed when instead a single hetero-dimer with significant size-mismatched AuNRs was investigated (Figure 5e-f). The single-particle scattering spectra recorded with LCP and RCP light (Figure 5e) as well as the resulting CDS spectrum (Figure 5f) clearly reveal the presence of the lower energy bonding mode that is no longer dark, likely due to symmetry breaking as will be discussed in more detail below. Such symmetry breaking results in a bisignate CD lineshape, and based on 
the sign of the two peaks we conclude that this hetero-dimer is a (-)-enantiomer with a negative twist angle $\theta$. This type of heterogeneity, different enantiomers and homo- vs. hetero-dimers, is inaccessible by traditional ensemble CD spectroscopy. ${ }^{34}$

Calculated scattering and CDS spectra of this hetero-dimer (Figure 5g-h) based on the correlated SEM image agreed with the major spectral features observed in the single-particle experiment despite some noticeable discrepancies at shorter wavelengths where the optical components start to fail. A negative twist angle was required to match the sign of the experimental bisignate lineshape confirming that this hetero-dimer is indeed a (-)-enantiomer. The charge plots in Figure 5h furthermore confirm the anti-bonding and bonding nature of the higher and lower energy peaks, respectively. However, some disagreement is noted between the measured and calculated spectra. In particular, in the calculated spectra the bonding mode was not as pronounced and the relative intensity difference in the scattering spectra for LCP and RCP excitation was significantly less. Although we have included the glass substrate, ligand capping layers, in-plane averaged excitations, used an excessively large twist angle $\theta=-45^{\circ}$, and have explored many combinations of the structural dimensions of the AuNRs, no exact match to the experimental spectra could be achieved. A minor contribution from out-of-plane excitation with the $k$-vector normal to the substrate plane was likely in the experiments due to the nature of the total internal reflection excitation. ${ }^{57}$ Further FDTD simulations of an ideal homo-dimer comparing in- and out-of-plane excitations (Figure S9) revealed that the CDS contribution from out-of-plane excitation is less than $10 \%$ compared to the CDS generated by excitation along the dimer twist axis and thus has only little effect on the CDS lineshape. In addition to limitations arising from extracting three-dimensional structure from two-dimensional SEM images, the correction factor described in Figure 4 represents a wavelength-averaged value over the 610 - 
$850 \mathrm{~nm}$ working range of the quarter-waveplate. In the $580-610 \mathrm{~nm}$ spectral range significant deviations from the simulations are observed in Figure $5 \mathrm{f}$ as to the quarter-wave length failed to produce circularly polarized light, resulting in increased artificial CDS due to linear dichroism.

The bisignate CDS lineshapes observed for the hetero-dimer sample (Figure $5 \mathrm{f}$ and $5 \mathrm{~h}$ ) were largely the result of symmetry breaking caused by size mismatch and an increased twist angle. To quantify these two factors, we again consider the ideal homo-dimer discussed in Figure 2 and first introduce size mismatch by increasing both the width and length of one AuNR by $20 \%$ so that the aspect ratio and hence resonance wavelength remain constant. The simulated homo-dimer and hetero-dimer absorption CD and CDS spectra (Figures 6a and 6b) reveal that, compared to the homo-dimer, the absorption CD increases for the bonding mode of the heterodimer and the previously unobserved CDS signal at this resonance is now visible. The mode assignment for both the homo- and hetero-dimers is given in Figure S10, which shows the corresponding absorption and scattering spectra and surface charge plots. The CDS intensity at the bonding mode originates from the fact that the two anti-parallel dipoles no longer completely cancel each other due to the size mismatch of the two AuNRs. ${ }^{51}$ We additionally performed simulations of a size-mismatched hetero-dimer with one AuNR 20\% smaller and observed the same bisignate CDS spectrum (Figure S11).

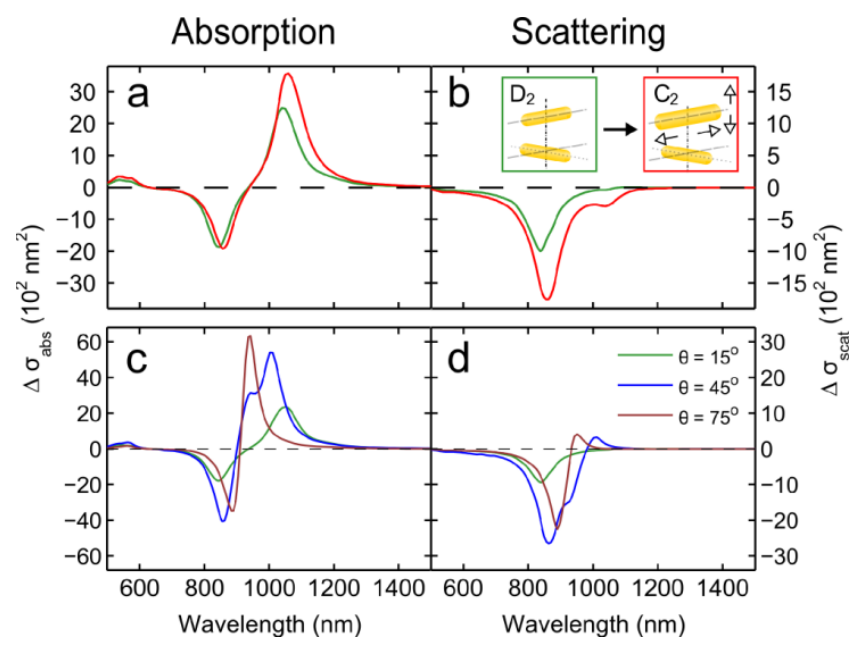


Figure 6. FDTD simulations showing the effects of symmetry-breaking on the CD and CDS spectrum of AuNR dimers. Calculated absorption CD (a) and CDS (b) spectra of a twisted AuNR homo-dimer (green line) vs. a hetero-dimer (red line). The homo-dimer dimensions were the same as in Figure 1, while a size mismatch was introduced by increasing both width and length of the second AuNR by 20\%. Varying width and length equally ensured that the aspect ratio was kept constant and therefore the resonance energy did not change. The corresponding linear absorption and scattering spectra as well as surface charge distributions are given in Figure S10. Calculated absorption (c) and scattering (d) spectra of homo-dimers with three distinct twist angles of $15^{\circ}$ (green line), $45^{\circ}$ (blue line) and $75^{\circ}$ (maroon line). Apart from the twist angle the simulation parameters were the same as in Figure 2.

A significantly larger twist angle of the AuNR dimer results in a bisignate lineshape of the CDS. Figures 6c and 6d illustrate the absorption CD and CDS spectra of an ideal homodimer for twist angles of $\theta=15^{\circ}, 45^{\circ}$, and $75^{\circ}$. As the twist angle is increased the bonding mode gains strength in both the absorption CD and CDS spectra. With significant excitation of the bonding mode at large twist angles, the CDS spectrum now shows a bisignate lineshape. Symmetry breaking due to the presence of the substrate appears to have a minor effect for this particular system (Figure S12). The simulations in Figure 6 considering simplified AuNR dimer geometries confirm that both a size mismatch and an increased twist angle contribute to the experimentally observed CDS lineshape of the hetero-dimer (Figure 5f).

\section{Conclusions}

We have demonstrated a detailed description of the CDS lineshape of AuNR dimers with emphasis on various forms of symmetry breaking, which naturally occurs in assembled chiral nanostructures. Single-particle CDS spectra were measured using a conventional dark-field microscope, made possible through a correction procedure for linear dichroism artifacts arising from imperfections in the incident circular polarization. Our experiments were complimented with simulations to disentangle, separately for absorption and scattering, the effects of symmetry breaking on the scattering and CDS spectra of twisted AuNR dimers. We found that the bisignate 
CDS lineshape reported based on ensemble extinction spectroscopy for AuNR dimers with the dimensions and geometries in this and previous studies ${ }^{34}$ is only observed for strongly asymmetric hetero-dimers. Our single-particle CDS method can be readily adapted to numerous other chiral nanoparticle systems, exemplified by twisted nanoribbons, ${ }^{16}$ as the field of chiral nanomaterials continues to rapidly expand and requires a detailed understanding between

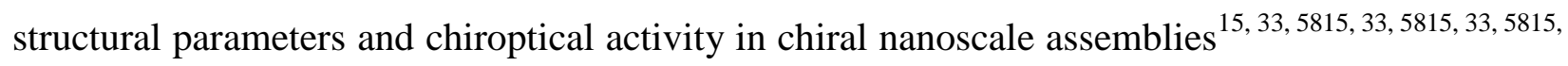
$33,5815,33,58$

\section{Methods}

\section{Nanoparticle Synthesis and Dimer Preparation}

Single AuNRs were purchased from NanoPartz with nominal dimensions of $22 \times 86 \mathrm{~nm}$. AuNR dimers were prepared as described in Ma et al. using a citrate linker molecule. ${ }^{34}$ Briefly, AuNRs with an aspect ratio of 2.1 (average lengths and diameters of $63.6 \mathrm{~nm}$ and $22.0 \mathrm{~nm}$ ), as verified by transmission electron microscopy (TEM), were prepared by a standard seeded growth method. The dimer formation was initiated through the addition of sodium citrate solution to the nanoparticle dispersion causing the AuNRs to oligomerize. The reaction was halted by drop casting the solution onto a substrate approximately five minutes after the reaction was initiated, followed by drying with ultra-pure $\mathrm{N}_{2}$ gas. The glass substrates were indexed for correlated SEM imaging ${ }^{59}$ with a FEI Quanta 400 ESEM FEG using an electron beam energy of $15 \mathrm{keV}$. SEM characterization revealed that no dimers with their twist axis perpendicular to the substrate were observed. Previous characterization of the same AuNR dimer samples using TEM tomography revealed an interparticle gap of $7.1 \mathrm{~nm}$ and a twist angle of $11^{0.34}$

\section{Dark-Field Spectroscopy}


The single-particle scattering experiments were performed using a typical dark-field microscope setup. As shown in Figure 3a an inverted microscope (Zeiss AxioObserver m1) was used to measure the CDS of single nanoparticles. Unpolarized light from a tungsten-halogen lamp was passed through a linear polarizer (ThorLabs, LPVIS100) followed by a quarter-wave plate (Edmund Optics, \#65-919, working range $600 \mathrm{~nm}-800 \mathrm{~nm}$ ) with axes oriented $\pm 45^{\circ}$ relative to one another to generate LCP or RCP light. An oil immersion condenser (Zeiss, NA = 1.4) was used to pass high angle incident light to the sample interface where total internal reflection occurred generating an evanescent wave which excited the nanoparticles. Scattered light was collected with a 50X objective (Zeiss NA $=0.8$ ) and passed through a slit aperture

towards a hyperspectral detection system, described previously. ${ }^{60}$ For each measurement day, the polarizer and waveplate orientations were adjusted by optimizing the circular polarized incident light using the bright-field microscope option while monitoring the lamp spectrum. This procedure yielded the same functional form of the correction factor (Scheme S3), but magnitude and phase varied slightly.

\section{Simulations}

A commercial simulator, Lumerical, based on the FDTD method was used to perform the calculations. The AuNRs were modeled as hemispherically-capped cylinders with the bulk metal dielectric function adopted from Johnson and Christy. ${ }^{61}$ The minimum mesh size was set to 0.5 nm to ensure convergence. An additional 4-nm-thick dielectric layer with a refractive index $n_{\text {layer }}$ $=2.2$ was included in the model to account for the surfactants surrounding the chemicallysynthesized AuNRs used in the experiments. ${ }^{62}$ The schematic diagrams of the FDTD model with and without a glass substrate as well as additional details are provided in Figures S1 and S4. To approximate total internal reflection excitation, where evanescent waves propagate along the 
substrate, the $k$-vector of the simulated excitation was assumed to be parallel to the substrate. An orthogonal $k$-vector for possibly minor out-of-plane excitation resulted in a negligible CDS response (Figure S9).

\section{Supporting Information}

An analytical model describing the excitation polarization, the resulting CDS artifacts, our implemented correction procedure for said artifacts, and further details regarding simulated results are contained in the supporting information. The Supporting Information is available free of charge on the ACS Publications website at DOI: 10.1021/acsphotonics.XXXXXXX.

\section{Acknowledgements}

This work was funded by the Robert A. Welch Foundation (C-1664), the Army Research Office (MURI W911NF-12-1-0407) and National Science Foundation (CHE1507745). K. W. S. and J. O. acknowledge that this material is based upon work supported by the National Science Foundation Graduate Research Fellowship Program (0940902). N.A.K. thanks NSF CBET and DMR directorates for grants that partially supported this project.

\section{References}

1. Wagniere, G. H., On Chirality and the Universal Asymmetry: Reflections on Image and Mirror Image. VHCA-Wiley-VCH: Zürich, Switzerland, 2007.

2. $\quad$ Yoon, T. P.; Jacobsen, E. N. Privileged Chiral Catalysts. Science 2003, 299, 1691-1693.

3. Pfaltz, A.; Drury, W. J. Design of Chiral Ligands for Asymmetric Catalysis: From C2Symmetric P,P- and N,N-Ligands to Sterically and Electronically Nonsymmetrical P,N-Ligands. Proc. Natl. Acad. Sci. U.S.A. 2004, 101, 5723-5726.

4. $\quad$ Bada, J. L. Origins of Homochirality. Nature 1995, 374, 594-595.

5. Sharma, V.; Crne, M.; Park, J. O.; Srinivasarao, M. Structural Origin of Circularly Polarized Iridescence in Jeweled Beetles. Science 2009, 325, 449-451.

6. Smith, S. W. Chiral Toxicology: It's the Same Thing... Only Different. Toxicol. Sci. 2009, 110, 4-30.

7. $\quad$ Berova, N.; Nakanishi, K.; Woody, R., Circular Dichroism: Principles and Applications. John Wiley \& Sons: New York, 2000.

8. Saxena, V. P.; Wetlaufer, D. B. A New Basis for Interpreting the Circular Dichroic Spectra of Proteins. Proc. Natl. Acad. Sci. U.S.A. 1971, 68, 969-972. 
9. Tamaru, S.-i.; Shinkai, S.; Khasanov, A. B.; Bell, T. W. Circular Dichroism Readout of Sugar Recognition in the Cleft of a Fused-Pyridine Receptor. Proc. Natl. Acad. Sci. USA 2002, 99, 4972-4976.

10. Pettegrew, J. W.; Miles, D. W.; Eyring, H. Circular Dichroism of Adenosine Dinucleotides. Proc. Natl. Acad. Sci. USA 1977, 74, 1785-1788.

11. Caldwell, D. J.; Eyring, H., The Theory of Optical Activity. Wiley-Interscience: 1971. 12. Auguié, B.; Alonso-Gómez, J. L.; Guerrero-Martínez, A. s.; Liz-Marzán, L. M. Fingers Crossed: Optical Activity of a Chiral Dimer of Plasmonic Nanorods. J. Phys. Chem. Lett. 2011, 2, 846-851.

13. Bustamante, C.; Tinoco, I.; Maestre, M. F. Circular Differential Scattering Can Be an Important Part of the Circular Dichroism of Macromolecules. Proc. Natl. Acad. Sci. USA 1983, 80, 3568-3572.

14. Gansel, J. K.; Thiel, M.; Rill, M. S.; Decker, M.; Bade, K.; Saile, V.; von Freymann, G.; Linden, S.; Wegener, M. Gold Helix Photonic Metamaterial as Broadband Circular Polarizer. Science 2009, 325, 1513-1515.

15. Kuzyk, A.; Schreiber, R.; Fan, Z.; Pardatscher, G.; Roller, E.-M.; Hogele, A.; Simmel, F. C.; Govorov, A. O.; Liedl, T. DNA-Based Self-Assembly of Chiral Plasmonic Nanostructures with Tailored Optical Response. Nature 2012, 483, 311-314.

16. Yeom, J.; Yeom, B.; Chan, H.; Smith, K. W.; Dominguez-Medina, S.; Bahng, J. H.; Zhao, G.; Chang, W.-S.; Chang, S.-J.; Chuvilin, A.; Melnikau, D.; Rogach, A. L.; Zhang, P.; Link, S.; Král, P.; Kotov, N. A. Chiral Templating of Self-Assembling Nanostructures by Circularly Polarized Light. Nat. Mater. 2015, 14, 6-72.

17. Chandra, M.; Dowgiallo, A.-M.; Knappenberger, K. L. Magnetic Dipolar Interactions in Solid Gold Nanosphere Dimers. J. Am. Chem. Soc. 2012, 134, 4477-4480.

18. Guerrero-Martínez, A.; Auguié, B.; Alonso-Gómez, J. L.; Džolić, Z.; Gómez-Graña, S.; Žinić, M.; Cid, M. M.; Liz-Marzán, L. M. Intense Optical Activity from Three-Dimensional Chiral Ordering of Plasmonic Nanoantennas. Angew. Chem. Int. Ed. 2011, 50, 5499-5503. 19. Li, Z.; Zhu, Z.; Liu, W.; Zhou, Y.; Han, B.; Gao, Y.; Tang, Z. Reversible Plasmonic Circular Dichroism of Au Nanorod and DNA Assemblies. J. Am. Chem. Soc. 2012, 134, 33223325.

20. Shen, X.; Asenjo-Garcia, A.; Liu, Q.; Jiang, Q.; García de Abajo, F. J.; Liu, N.; Ding, B. Three-Dimensional Plasmonic Chiral Tetramers Assembled by DNA Origami. Nano Lett. 2013, 13, 2128-2133.

21. Valev, V. K.; Baumberg, J. J.; De Clercq, B.; Braz, N.; Zheng, X.; Osley, E. J.; Vandendriessche, S.; Hojeij, M.; Blejean, C.; Mertens, J.; Biris, C. G.; Volskiy, V.; Ameloot, M.; Ekinci, Y.; Vandenbosch, G. A. E.; Warburton, P. A.; Moshchalkov, V. V.; Panoiu, N. C.; Verbiest, T. Nonlinear Superchiral Meta-Surfaces: Tuning Chirality and Disentangling NonReciprocity at the Nanoscale. Adv. Mater. 2014, 26, 4074-4081.

22. Ben-Moshe, A.; Wolf, S. G.; Sadan, M. B.; Houben, L.; Fan, Z.; Govorov, A. O.; Markovich, G. Enantioselective Control of Lattice and Shape Chirality in Inorganic Nanostructures Using Chiral Biomolecules. Nat Commun 2014, 5, 4302.

23. Biswas, S.; Liu, X.; Jarrett, J. W.; Brown, D.; Pustovit, V.; Urbas, A.; Knappenberger, K. L.; Nealey, P. F.; Vaia, R. A. Nonlinear Chiro-Optical Amplification by Plasmonic Nanolens Arrays Formed Via Directed Assembly of Gold Nanoparticles. Nano Lett. 2015, 15, 1836-1842.

24. Narushima, T.; Okamoto, H. Circular Dichroism Nano-Imaging of Two-Dimensional Chiral Metal Nanostructures. PCCP 2013, 15, 13805-13809. 
25. Pendry, J. B. A Chiral Route to Negative Refraction. Science 2004, 306, 1353-1355.

26. Kuwata-Gonokami, M.; Saito, N.; Ino, Y.; Kauranen, M.; Jefimovs, K.; Vallius, T.;

Turunen, J.; Svirko, Y. Giant Optical Activity in Quasi-Two-Dimensional Planar Nanostructures. Phys. Rev. Lett. 2005, 95, 227401.

27. Zhao, Y.; Belkin, M. A.; Alù, A. Twisted Optical Metamaterials for Planarized Ultrathin Broadband Circular Polarizers. Nat. Commun. 2012, 3, 870.

28. Hendry, E.; Carpy, T.; Johnston, J.; Popland, M.; Mikhaylovskiy, R. V.; Lapthorn, A. J.;

Kelly, S. M.; Barron, L. D.; Gadegaard, N.; Kadodwala, M. Ultrasensitive Detection and Characterization of Biomolecules Using Superchiral Fields. Nat. Nanotech. 2010, 5, 783-787.

29. Maoz, B. M.; Chaikin, Y.; Tesler, A. B.; Bar Elli, O.; Fan, Z.; Govorov, A. O.;

Markovich, G. Amplification of Chiroptical Activity of Chiral Biomolecules by Surface Plasmons. Nano Lett. 2013, 13, 1203-1209.

30. García-Etxarri, A.; Dionne, J. A. Surface-Enhanced Circular Dichroism Spectroscopy Mediated by Nonchiral Nanoantennas. Phys. Rev. B 2013, 87, 235409.

31. Hentschel, M.; Schäferling, M.; Weiss, T.; Liu, N.; Giessen, H. Three-Dimensional Chiral Plasmonic Oligomers. Nano Lett. 2012, 12, 2542-2547.

32. Yin, X.; Schäferling, M.; Metzger, B.; Giessen, H. Interpreting Chiral Spectra: The Plasmonic Born-Kuhn Model. Nano Lett. 2013, 13, 6238-6243.

33. Liu, M.; Powell, D. A.; Shadrivov, I. V.; Lapine, M.; Kivshar, Y. S. Spontaneous Chiral Symmetry Breaking in Metamaterials. Nat Commun 2014, 5, 4441.

34. Ma, W.; Kuang, H.; Wang, L.; Xu, L.; Chang, W.-S.; Zhang, H.; Sun, M.; Zhu, Y.; Zhao, Y.; Liu, L.; Xu, C.; Link, S.; Kotov, N. A. Chiral Plasmonics of Self-Assembled Nanorod Dimers. Sci. Rep. 2013, 3, 1934

35. Lan, X.; Lu, X.; Shen, C.; Ke, Y.; Ni, W.; Wang, Q. Au Nanorod Helical Superstructures with Designed Chirality. J. Am. Chem. Soc. 2014, 137, 457-462.

36. Shemer, G.; Krichevski, O.; Markovich, G.; Molotsky, T.; Lubitz, I.; Kotlyar, A. B. Chirality of Silver Nanoparticles Synthesized on DNA. J. Am. Chem. Soc. 2006, 128, 1100611007.

37. Querejeta-Fernández, A.; Chauve, G.; Methot, M.; Bouchard, J.; Kumacheva, E. Chiral Plasmonic Films Formed by Gold Nanorods and Cellulose Nanocrystals. J. Am. Chem. Soc. 2014, 136, 4788-4793.

38. Brongersma, M. L.; Halas, N. J.; Nordlander, P. Plasmon-Induced Hot Carrier Science and Technology. Nat Nano 2015, 10, 25-34.

39. Novotny, L.; van Hulst, N. Antennas for Light. Nat Photon 2011, 5, 83-90.

40. $\quad$ Olson, J.; Dominguez-Medina, S.; Hoggard, A.; Wang, L.-Y.; Chang, W.-S.; Link, S. Optical Characterization of Single Plasmonic Nanoparticles. Chem. Soc. Rev. 2014, 44, 40-57. 41. Gennaro, S. D.; Sonnefraud, Y.; Verellen, N.; Van Dorpe, P.; Moshchalkov, V. V.; Maier, S. A.; Oulton, R. F. Spectral Interferometric Microscopy Reveals Absorption by Individual Optical Nanoantennas from Extinction Phase. Nat Commun 2014, 5, 3748.

42. $\quad$ Arbouet, A.; Christofilos, D.; Del Fatti, N.; Vallee, F.; Huntzinger, J. R.; Arnaud, L.; Billaud, P.; Broyer, M. Direct Measurement of the Single-Metal-Cluster Optical Absorption. Phys. Rev. Lett. 2004, 93, 127401.

43. Lindfors, K.; Kalkbrenner, T.; Stoller, P.; Sandoghdar, V. Detection and Spectroscopy of Gold Nanoparticles Using Supercontinuum White Light Confocal Microscopy. Phys. Rev. Lett. 2004, 93, 037401. 
44. Boyer, D.; Tamarat, P.; Maali, A.; Lounis, B.; Orrit, M. Photothermal Imaging of Nanometer-Sized Metal Particles among Scatterers. Science 2002, 297, 1160-1163.

45. Chang, W.-S.; Ha, J. W.; Slaughter, L. S.; Link, S. Plasmonic Nanorod Absorbers as Orientation Sensors. Proc. Natl. Acad. Sci. U.S.A. 2010, 107, 2781-2786.

46. Nie, Z.; Petukhova, A.; Kumacheva, E. Properties and Emerging Applications of SelfAssembled Structures Made from Inorganic Nanoparticles. Nat. Nanotech. 2010, 5, 15-25.

47. $\quad$ Ringe, E.; Sharma, B.; Henry, A.-I.; Marks, L. D.; Van Duyne, R. P. Single Nanoparticle Plasmonics. PCCP 2013, 15, 4110-4129.

48. Lu, X.; Wu, J.; Zhu, Q.; Zhao, J.; Wang, Q.; Zhan, L.; Ni, W. Circular Dichroism from Single Plasmonic Nanostructures with Extrinsic Chirality. Nanoscale 2014, 6, 14244-14253.

49. Jarrett, J. W.; Herbert, P. J.; Dhuey, S.; Schwartzberg, A. M.; Knappenberger, K. L. Chiral Nanostructures Studied Using Polarization-Dependent Noles Imaging. J. Phys. Chem. A 2014, 118, 8393-8401.

50. Prodan, E.; Radloff, C.; Halas, N. J.; Nordlander, P. A Hybridization Model for the Plasmon Response of Complex Nanostructures. Science 2003, 302, 419-422.

51. Jain, P. K.; Eustis, S.; El-Sayed, M. A. Plasmon Coupling in Nanorod Assemblies: Optical Absorption, Discrete Dipole Approximation Simulation, and Exciton-Coupling Model. $J$. Phys. Chem. B 2006, 110, 18243-18253.

52. Smith, D. Y. Superconvergence and Sum Rules for the Optical Constants: Natural and Magneto-Optical Activity. Phys. Rev. B 1976, 13, 5303-5315.

53. Sönnichsen, C.; Geier, S.; Hecker, N. E.; von Plessen, G.; Feldmann, J.; Ditlbacher, H.; Lamprecht, B.; Krenn, J. R.; Aussenegg, F. R.; Chan, V. Z.-H.; Spatz, J. P.; Möller, M. Spectroscopy of Single Metallic Nanoparticles Using Total Internal Reflection Microscopy. Appl. Phys. Lett. 2000, 77, 2949-2951.

54. Tang, Y.; Cook, T. A.; Cohen, A. E. Limits on Fluorescence Detected Circular Dichroism of Single Helicene Molecules. J. Phys. Chem. A 2009, 113, 6213-6216.

55. Disch, R. L.; Sverdlik, D. I. Apparent Circular Dichroism of Oriented Systems. Anal. Chem. 1969, 41, 82-86.

56. Fan, Z.; Govorov, A. O. Helical Metal Nanoparticle Assemblies with Defects: Plasmonic Chirality and Circular Dichroism. J. Phys. Chem. C 2011, 115, 13254-13261.

57. Azzam, R. M. A. Circular and near-Circular Polarization States of Evanescent Monochromatic Light Fields in Total Internal Reflection. Appl. Opt. 2011, 50, 6272-6276.

58. Cui, Y.; Kang, L.; Lan, S.; Rodrigues, S.; Cai, W. Giant Chiral Optical Response from a Twisted-Arc Metamaterial. Nano Lett. 2014, 14, 1021-1025.

59. Slaughter, L.; Chang, W.-S.; Link, S. Characterizing Plasmons in Nanoparticles and Their Assemblies with Single Particle Spectroscopy. J. Phys. Chem. Lett. 2011, 2, 2015-2023.

60. Byers, C. P.; Hoener, B. S.; Chang, W.-S.; Yorulmaz, M.; Link, S.; Landes, C. F. SingleParticle Spectroscopy Reveals Heterogeneity in Electrochemical Tuning of the Localized Surface Plasmon. J. Phys. Chem. B 2014, 118, 14047-14055.

61. Johnson, P. B.; Christy, R. W. Optical Constants of the Noble Metals. Phys. Rev. B 1972, 6, 4370-4379.

62. Hoggard, A.; Wang, L.-Y.; Ma, L.; Fang, Y.; You, G.; Olson, J.; Liu, Z.; Chang, W.-S.; Ajayan, P. M.; Link, S. Using the Plasmon Linewidth to Calculate the Time and Efficiency of Electron Transfer between Gold Nanorods and Graphene. ACS Nano 2013, 7, 11209-11217. 